\title{
THE FATOU-ZYGMUND PROPERTY FOR SIDON SETS ${ }^{1}$
}

\author{
BY S. W. DRURY
}

Communicated by Kenneth A. Ross, October 10, 1973

A subset $X$ of a discrete abelian group $G$ is said to be a Sidon set if every bounded complex-valued function on $X$ is the restriction to $X$ of a Fourier-Stieltjes transform on $G$. In this article we give an affirmative answer to a question of J.-E. Bjork [1] and N. Th. Varopoulos [6].

THEOREM 1. Let $X$ be a symmetric Sidon subset of $G$ not containing $0_{G}$. Then every bounded hermitian function on $X$ is the restriction to $X$ of $a$ positive-definite function on $G$.

In the terminology of Edwards, Hewitt and Ross [2], the set $X$ has the Fatou-Zygmund property. We refer the reader to this article and to Ross [7] for a deeper understanding of the content of Theorem 1. The proof of Theorem 1 uses the technique of [3] but the presentation we give is akin to that of [4]. Unexplained notations and definitions may be found in [5].

For technical reasons we should like $X$ to be a finite set. Thus we shall actually prove the following result.

THEOREM 2. For all $\alpha(0<\alpha \leqq 1)$ there is a constant $C(\alpha)$ such that for every finite symmetric Sidon $(\alpha)$ subset $X$ of $G$ not containing $0_{G}$ and every hermitian function $\phi$ on $X$ with $\|\phi\|_{\infty} \leqq 1$, there exists $\mu$ a positive measure on $\hat{G}$ with $\|\mu\|_{M} \leqq C(\alpha)$ such that $\left.\hat{\mu}\right|_{X}=\phi$.

It is an easy consequence of Theorem 2 that the analogous statement with the word finite deleted holds. Thus Theorem 1 follows from Theorem 2. From now on let $X$ be as in Theorem 2 .

We fix $n$ to be an even integer greater than or equal to four and define $\Omega$ to be the finite group of hermitian mappings from $X$ to the complex $n$th roots of unity under pointwise multiplication. If $U$ denotes the set of

AMS (MOS) subject classifications (1970). Primary 43A25, 43A35.

Key words and phrases. Sidon set, Fatou-Zygmund set, interpolation set, FourierStieltjes transform, positive definite function.

1 The research for this article was supported by the National Research Council of Canada. 
all hermitian functions of $X$ into the closed unit disc we have

$$
U \subseteq \sec (\pi / n) \cdot \operatorname{co}(\Omega)
$$

where $\operatorname{co}(\Omega)$ denotes the real-affine convex hull of $\Omega$. This is not true if $n=2$ or if $n$ is odd and $X$ contains elements of order two.

The next lemma is a modification of the convolution device lemma of [4].

Lemma 3. There exist functions $g, g^{*}, g^{+}$and $g^{-}$on $G \times \Omega$ having the following properties

(1) $g=g^{+}-g^{-}, g^{*}=g^{+}+g^{-}$,

(2) $g_{\omega}^{ \pm}$is positive definite on $G \forall \omega \in \Omega$,

(3) $g(x, \omega)=\omega(x) \forall \omega \in \Omega, \forall x \in X$,

(4) $\left\|g_{\omega}^{ \pm}\right\|_{B(G)} \leqq \alpha^{-2} \forall \omega \in \Omega$,

(5) $\left\|g_{x}^{*}\right\|_{A(\Omega)} \leqq \alpha^{-2} \forall x \in G$.

Proof. Since $X$ is Sidon $(\alpha)$ there exist functions $f_{\omega}(\omega \in \Omega)$ on $G$ such that $f_{\omega}(x)=\omega(x) \forall \omega \in \Omega, \forall x \in X ;\left\|f_{\omega}\right\|_{B(G)} \leqq \alpha^{-1} \forall \omega \in \Omega$. We may assume that each $f_{\omega}$ is hermitian on $G$ for if not it suffices to throw away its skew-hermitian part. Thus we may write $f_{\omega}=f_{\omega}^{+}-f_{\omega}^{-}$where $f_{\omega}^{ \pm}$is positive definite on $G$. Now define

$$
g^{ \pm \pm}(x, \omega)=\int f^{ \pm}\left(x, \omega \lambda^{-1}\right) f^{ \pm}(x, \lambda) d \eta(\lambda)
$$

where $\eta$ is the invariant probability measure on $\Omega$. We set $g^{+}=g^{++}+g^{--}$, $g^{-}=g^{+-}+g^{-+}, g=g^{+}-g^{-}$and $g^{*}=g^{+}+g^{-}$. Conditions (1)-(3) are easily checked and (4)-(5) follow as in [4].

Let $H$ denote the dual group of $\Omega$, that is, the $Z(n)$-module generated by $X$ and the relations $x+(-x)=0(x \in X)$. The negation mapping on $X$ induces inversion on $\Omega$

$$
\omega(-x)=\overline{\omega(x)}=\omega^{-1}(x)
$$

which in turn induces negation on $H$. The natural injection $j$ of $X$ into $H$ given by $\langle j(x), \omega\rangle=\omega(x)$ thus satisfies $j(-x)=-j(x)$. A finite subset $Y$ of a discrete abelian group $F$ is said to be symmetric $n$-independent if and only if

(a) $Y$ is symmetric.

(b) If $m: Y \rightarrow Z$ and $\sum_{y \in Y} m(y) \cdot y=0_{F}$ then $m(y)-m(-y) \equiv 0 \bmod n$ for all $y \in Y$ and $m(y) \equiv 0 \bmod 2$ for all $y \in Y$ with $2 y=0_{F}$. It is easy to prove that the subsets $j(X)$ and $\operatorname{graph}(j)=\{(x, j(x)) ; x \in X\}$ are symmetric $n$-independent in $H$ and $G \times H$ respectively. 
LEMMA 4. Let $0<\varepsilon \leqq 1$ and suppose that $Y$ is a symmetric $n$-independent subset of $F$. There exist functions $p^{+}, p^{-}, p^{e}$ and $p^{o}$ on $F$ such that

(1') $p^{+}=p^{e}+p^{o}, p^{-}=p^{e}-p^{o}$;

(2') $p^{ \pm}$is positive definite on $F$;

(3') $p^{o}(y)=1 / 2 \varepsilon \forall y \in Y$;

(4') $\left\|p^{ \pm}\right\|_{B\left(F^{\prime}\right)}=1$;

$\left(5^{\prime}\right)\left|p^{e}(y)\right| \leqq \varepsilon^{2} \forall y \in F \backslash\left\{0_{F}\right\}$.

The letters $e$ and o stand for even and odd.

Proof. Let $Q$ denote the quotient of $Y$ induced by the equivalence relation $y_{1} \sim y_{2}$ if and only if either $y_{1}=y_{2}$ or $y_{1}=-y_{2}$. For $q \in Q$ and $\chi \in \hat{F}$ we define

$$
a_{q}^{ \pm}(\chi)=1 \pm \frac{\varepsilon}{2} \sum_{y \in q} \chi(y)
$$

and the cosine Riesz products $p^{ \pm}$are defined by

$$
\left(p^{ \pm}\right)^{\wedge}(\chi)=\prod_{q \in Q} a_{q}^{ \pm}(\chi)
$$

The definition of $p^{e}$ and $p^{o}$ is given by $\left(1^{\prime}\right)$. The verification of $\left(2^{\prime}\right),\left(3^{\prime}\right)$ and $\left(4^{\prime}\right)$ is routine-see for example $\left[5\right.$, p. 124]. To prove $\left(5^{\prime}\right)$ we establish by direct calculation that

$$
p^{e}(z)=\sum\left(\frac{1}{2} \varepsilon\right)^{\operatorname{card}(R)} C_{R}(z)
$$

where the summation is over all even subsets $R$ of $Q$ and $C_{R}(z)$ is the number of partial section maps $y: R \rightarrow Y$ for which $z=\sum_{q \in R} y(q)$. The definition of symmetric $n$-independence ensures that for each fixed $z, C_{R}(z)$ is nonzero for at most one value of $R$. Thus

$$
\left|p^{e}(z)\right| \leqq \sup \left(\frac{1}{2} \varepsilon\right)^{\operatorname{card}(R)} C_{R}(z) .
$$

Since $\operatorname{card}(q) \leqq 2$ for all $q$ in $Q$ it follows that $C_{R}(z) \leqq 2^{\operatorname{card}(R)}$. Clearly $C_{\varnothing}(z)=0$ for $z \neq 0_{F}$. Recalling that the supremum is only over sets of even cardinality we have $\left(5^{\prime}\right)$.

Proof of Theorem 2. We use the notation of Lemmas 3 and 4 where $Y=\operatorname{graph}(j)$ and $F=G \times H$. We define

$$
s(x, \omega)=\int\left[\left(p^{+}\right)^{\wedge}\left(x, \omega \lambda^{-1}\right) g^{+}(x, \lambda)+\left(p^{-}\right)^{\wedge}\left(x, \omega \lambda^{-1}\right) g^{-}(x, \lambda)\right] d \eta(\lambda)
$$

where ${ }^{\wedge}$ denotes the Fourier transform in the $\Omega, H$ duality only. By (2) and $\left(2^{\prime}\right), s_{\omega}$ is positive definite in $G$ for each $\omega$ in $\Omega$. By (4) and $\left(4^{\prime}\right)$, 
$\left\|s_{\omega}\right\|_{B(G)} \leqq 2 \alpha^{-2} \forall \omega \in \Omega$. Now we rewrite $s$.

$$
\begin{aligned}
s(x, \omega) & =\int\left(p^{o}\right)^{\wedge}\left(x, \omega \lambda^{-1}\right) g(x, \lambda) d \eta(\lambda)+\int\left(p^{e}\right)^{\wedge}\left(x, \omega \lambda^{-1}\right) g^{*}(x, \lambda) d \eta(\lambda) \\
& =s^{o}(x, \omega)+s^{e}(x, \omega) .
\end{aligned}
$$

By (3) and ( $\left.3^{\prime}\right), s^{o}(x, \omega)=\frac{1}{2} \varepsilon \omega(x) \forall \omega \in \Omega, \forall x \in X$. By (5), (5') and since $0_{G} \notin X,\left|s^{e}(x, \omega)\right| \leqq \varepsilon^{2} \alpha^{-2} \forall \omega \in \Omega, \forall x \in X$. Hence

$$
\left|s(x, \omega)-\frac{1}{2} \varepsilon \omega(x)\right| \leqq \varepsilon^{2} \alpha^{-2} \quad \forall \omega \in \Omega, \forall x \in X .
$$

Now by real-affine convexity and the condition $(*)$ we have that for each element $\phi$ of $U$ there exists a positive measure $\mu$ on $\hat{G}$ such that

$$
\begin{aligned}
\|\mu\|_{M} & \leqq 4 \varepsilon^{-1} \alpha^{-2} \sec (\pi / n), \\
\left\|\left.\hat{\mu}\right|_{X}-\phi\right\|_{\infty} & \leqq 2 \varepsilon \alpha^{-2} \sec (\pi / n) .
\end{aligned}
$$

Now select $\varepsilon=\frac{1}{4} \alpha^{2} \cos (\pi / n)$. Since $\left.\hat{\mu}\right|_{X}-\phi$ is again hermitian on $X$, Theorem 2 follows by iteration. The constant $C(\alpha)$ may be taken to be $32 \alpha^{-4}$.

\section{REFERENCES}

1. L.-A. Lindahl and F. Poulsen, Thin sets in harmonic analysis, Problem 5.5, p. 67, Lecture Notes in Pure and Appl. Math., Dekker, New York, 1971.

2. R. E. Edwards, E. Hewitt and K. A. Ross, Lacunarity for compact groups. III, Studia Math. 44 (1972), 429-476.

3. S. W. Drury, Sur les ensembles de Sidon, C.R. Acad. Sci. Paris Sér. A-B 271 (1970), A162-A163. MR 42 \#6530.

4. - Unions of sets of interpolation, Conference on Harmonic Analysis, Maryland, Lecture Notes in Math., no. 266, Springer-Verlag, New York, 1972, pp. 2333.

5. W. Rudin, Fourier analysis on groups, Interscience Tracts in Pure and Appl. Math., no. 12, Interscience, New York, 1962. MR 27 \#2808.

6. N. Th. Varopoulos, Seminar on sets of interpolation, Mittag-Leffler Institute, 1969-70 (multilith).

7. K. A. Ross, Fatou-Zygmund sets, Proc. Cambridge Philos. Soc. 73 (1973), 57-65.

Department of Mathematics, McGill University, Montreal, Quebec, Canada 